\title{
An unusual case of shortness of breath
}

\section{S F Wan Muhammad Hatta',2, L Kandaswamy', C Gherman-Ciolac', J Mann ${ }^{1}$ and H N Buch ${ }^{1}$}

${ }^{1}$ New Cross Hospital, Wolverhampton, UK and 2Faculty of Medicine, Universiti Teknologi MARA, Sungai Buloh Campus, 47000 Sungai Buloh, Selangor, Malaysia
Correspondence should be addressed to S F Wan Muhammad Hatta

Email

sharifah.faradila@nhs.net

\section{Summary}

Myopathy is a well-known complication of hypercortisolism and commonly involves proximal lower-limb girdle. We report a rare case of Cushing's syndrome in a 60-year-old female presenting with significant respiratory muscle weakness and respiratory failure. She had history of rheumatoid arthritis, primary biliary cirrhosis and primary hypothyroidism and presented with weight gain and increasing shortness of breath. Investigations confirmed a restrictive defect with impaired gas transfer but with no significant parenchymatous pulmonary disease. Respiratory muscle test confirmed weakness of respiratory muscles and diaphragm. Biochemical and radiological investigations confirmed hypercortisolaemia secondary to a left adrenal tumour. Following adrenalectomy her respiratory symptoms improved along with an objective improvement in the respiratory muscle strength, diaphragmatic movement and pulmonary function test.

\section{Learning points:}

- Cushing's syndrome can present in many ways, a high index of suspicion is required for its diagnosis, as often patients present with only few of the pathognomonic symptoms and signs of the syndrome.

- Proximal lower-limb girdle myopathy is common in Cushing's syndrome. Less often long-term exposure of excess glucocorticoid production can also affect other muscles including respiratory muscle and the diaphragm leading to progressive shortness of breath and even acute respiratory failure.

- Treatment of Cushing's myopathy involves treating the underlying cause that is hypercortisolism. Various medications have been suggested to hinder the development of GC-induced myopathy, but their effects are poorly analysed.

\section{Background}

The symptoms and signs of Cushing's syndrome are secondary to long-term exposure to high level of glucocorticoids. A high index of suspicion is required for diagnosis as commonly patients present with only few of the pathognomonic symptoms and signs of the syndrome. Obesity, hypertension and glucose intolerance, which commonly occur secondary to hypercortisolism, are non-specific features and are common in background population. An important clue to the presence of excess glucocorticoid production is simultaneous development of several of these symptoms with increasing severity. Myopathy is one of the more common features of Cushing's syndrome, and we present an unusual presentation of myopathy in a patient of Cushing's syndrome.

\section{Case presentation}

A 60-years-old Caucasian female with a known history of rheumatoid arthritis, primary biliary cirrhosis and primary hypothyroidism presented to respiratory outpatient clinic in 2009 with the complaints of slow progressive dyspnoea on exertion, reduced effort tolerance and weight gain of 2 stones over the past 3 years. She denied cough, orthopnoea, paroxysmal 
nocturnal dyspnoea or chest pain. She was an ex-smoker with a smoking history of 35 pack-years. There was no history of exposure to any specific aero-allergens, industrial dusts or new pets and no family history of lung diseases. She received treatment in the form of oral steroids for a short period of time ( $<4$ weeks) in the past 3 years for her underlying illnesses. She had also received two intra-articular steroids injection during this time period.

\section{Investigation}

Throughout the period between 2009 and 2012, she was followed up in the respiratory clinic but no definite diagnosis could be arrived at. She underwent multiple investigations including chest radiographs, which were normal, spirometry which showed restrictive defect with impaired gas transfer (Table 1A) and high-resolution computed tomography (HRCT) which showed scattered small nodules, secondary to pulmonary involvement in rheumatoid arthritis, and early bronchiectasis affecting the mid and lower zones, with no evidence of interstitial lung disease or pulmonary embolism to explain the finding of restrictive defect on spirometry. An echocardiogram was normal with an ejection fraction of $55 \%$. In view of difficulty in obtaining a definite diagnosis, she had lung biopsy that showed mild fibrosis and non-specific chronic inflammation. A provisional diagnosis of pulmonary fibrosis was made, and she was commenced on a short therapeutic trial of prednisolone $40 \mathrm{mg}$ daily, which did not result in any improvement. Throughout the years, her condition deteriorated with worsening dyspnoea, proximal myopathy and further weight gain of two and half stones.

In early 2013, she presented to the Emergency Department with worsening dyspnoea, fever, cough productive of green sputum. Clinical examination revealed pulse rate of $100 \mathrm{bpm}$, blood pressure $160 / 80 \mathrm{mmHg}$, temperature $38^{\circ} \mathrm{C}$, respiratory rate of $30 / \mathrm{min}$ and oxygen

Table 1 Spirometry results pre and post surgery.

\begin{tabular}{|c|c|c|c|c|}
\hline Year & TLCO (\%) & FEV1 $(\%)$ & FVC $(\%)$ & FEV1/FVC (\%) \\
\hline \multicolumn{5}{|c|}{ (A) Pre surgery } \\
\hline 2010 & 57.3 & 73 & 77 & 94.8 \\
\hline 2011 & 57.1 & 73.5 & 78 & 94.2 \\
\hline 2012 & 32.2 & 56.3 & 62.2 & 90.5 \\
\hline 2013 & 48.4 & 63.1 & 66.7 & 94.6 \\
\hline \multicolumn{5}{|c|}{ (B) Post surgery } \\
\hline 2014 & 67 & 61 & 66 & 92.4 \\
\hline 2015 & 75 & 74 & 81 & 91.4 \\
\hline
\end{tabular}

Table 2 Plethysmography results pre and post surgery.

\begin{tabular}{|c|c|c|c|c|}
\hline Date & $\begin{array}{c}\text { MEP } \\
\text { predicted }\end{array}$ & $\begin{array}{l}\text { Normal } \\
\text { values }\end{array}$ & $\begin{array}{c}\text { MIP } \\
\text { predicted }\end{array}$ & $\begin{array}{l}\text { Normal } \\
\text { values }\end{array}$ \\
\hline \multicolumn{5}{|l|}{ (A) Pre-surgery } \\
\hline 2013 & 100 & $\begin{array}{c}>80 \mathrm{~cm} \text { of } \\
\text { water }\end{array}$ & 41 & $\begin{array}{c}>80 \mathrm{~cm} \text { of } \\
\text { water }\end{array}$ \\
\hline \multicolumn{5}{|l|}{ (B) Post surgery } \\
\hline 2014 & 112 & $\begin{array}{c}>80 \mathrm{~cm} \text { of } \\
\text { water }\end{array}$ & 89 & $\begin{array}{c}>80 \mathrm{~cm} \text { of } \\
\text { water }\end{array}$ \\
\hline
\end{tabular}

saturations of $80 \%$ on room air. Respiratory examination revealed bilateral coarse crackles bi-basally. Chest radiograph showed evidence of left basal consolidation. A diagnosis of severe community acquired pneumonia was made. She was started on broad-spectrum antibiotics and required admission to the intensive care unit for ventilatory support. Her condition improved and she was successfully extubated, but her symptoms of dyspnoea and reduced effort tolerance were persistent. During her admission, elevated liver enzymes led to an abdominal ultrasonography that showed fatty liver and incidental finding of significant reduction in the movement of both sides of diaphragm. This prompted further assessment of respiratory muscle function and pressure plethysmography showed a restrictive ventilatory failure with reduced maximum inspiratory and expiratory pressures (MIP and MEP), strongly suggestive of neuromuscular disease (Table 2A). Electromyogram, nerve conduction studies and antibodies for myasthenia gravis were normal.

Post discharge, she was clinically suspected to have Cushing's syndrome and finally seen by the endocrine team in clinic. Clinical examination revealed typical features of Cushing's syndrome, which was previously attributed to the previous use of steroids, in the form of abdominal obesity, supraclavicular and nape of the neck pad of fat, proximal muscle weakness, bruising and thinning of skin, bilateral leg oedema but no striae. Pulse rate was 72 beats/min (bpm), blood pressure $178 / 96 \mathrm{mmHg}$, temperature $37^{\circ} \mathrm{C}$, weight $76.4 \mathrm{~kg}$, BMI $28.8 \mathrm{~kg} / \mathrm{m}^{2}$ and oxygen saturation $98 \%$ on room air. Respiratory examination revealed coarse crepitation over bilateral lung bases. Neurological examination showed evidence of proximal muscle weakness of the upper and lower limbs with a grade of $3 / 5$, with preservation of deep tendon reflexes, normal temperature perception, position and vibratory sensation. Cardiovascular and abdominal examinations were unremarkable.

When seen in the endocrine clinic, she had DEXA scan which revealed osteopenia with a $T$ score of -2.3 in the lumbar spine (17\% decline in 3 years) and -1.8 at the neck of the left femur. Vertebral collapse was noted 
Myopathy in Cushing's syndrome
ID: 18-0074; July 2018 DOI: 10.1530/EDM-18-0074 at the level of T12. A probable diagnosis of Cushing's syndrome was made which was confirmed by endocrine testing and the mechanism identified as being non-ACTH dependent (Table 3). Blood investigations did not reveal any evidence of hypokalaemia or any acid-base disorder. A non-contrasted computed tomography scan with adrenal protocol identified a lipid-poor $2.6 \mathrm{~cm}$ diameter enhancing mass in the left adrenal gland consistent with an adrenal adenoma (Fig. 1). Lipid-poor adenomas would raise suspicion of a pheochromocytoma or an adrenocortical carcinoma. Clinical and laboratory investigations did not support the former possibility and the relatively modest size of the tumour with a long history over many years and absence of any other adverse imaging characteristics like extra-adrenal invasion or metastasis, made the latter an unlikely pre-surgical possibility.

\section{Treatment}

In October 2013, she underwent left laparoscopic adrenalectomy. Unfortunately, a full histology report was not able to be obtained due to the disintegration of the gland during extraction of the retrieval bag through a $10 \mathrm{~mm}$ port. Nevertheless, from limited histological assessment, it was confirmed to be a benign adrenal adenoma. Follow-up over the past 5 years has been reassuring with no evidence of persistent or recurrent pathology confirming the benign nature of the adenoma.

\section{Outcome and follow-up}

Post surgery, her blood pressure normalised, she lost $4 \mathrm{~kg}$ amount of weight and she showed significant regression of cushingoid features with an improvement in effort tolerance and mobility. Pressure plethysmography

Table 3 Blood investigation confirming the diagnosis of Cushing's syndrome.

\begin{tabular}{|c|c|c|}
\hline Investigations & Results & Normal range \\
\hline Overnight DST & 447 & $<50 \mathrm{nmol} / \mathrm{L}$ \\
\hline 24-h UFC & 502 & 0-130 nmol/L/24h \\
\hline ACTH & $<5$ & 7.2-63.3 ng/L \\
\hline $\mathrm{LH}$ & 10.4 & 5.2-61.9 IU/L \\
\hline FSH & 42.3 & 26.8-113.4 \\
\hline Oestrodiol & $<37$ & <37-103 pmol/L \\
\hline TSH, mU/L & 0.620 & \\
\hline Free thyroxine, pmol/L & 15 & \\
\hline Growth hormone & 0.2 & \\
\hline IGF-1 & 34.3 & \\
\hline
\end{tabular}

DST, dexamethasone suppression test; UFC, urinary free cortisol.

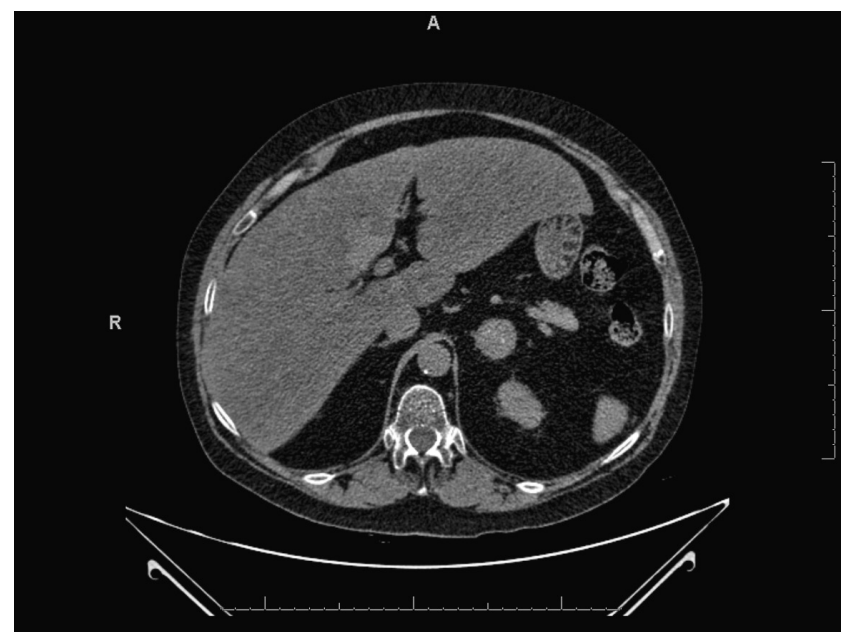

Figure 1

CT Abdomen showing a $2.6 \mathrm{~cm}$ mass within the left adrenal, with a Hounsfield Unit of $40 \mathrm{HU}$ on unenhanced scan. Right adrenal is normal.

showed marked improvement (Table 2B). Unfortunately her symptoms of joint pains and pruritus related to rheumatoid arthritis and primary biliary cirrhosis worsened, presumably related to a reduction in excessive amount of circulating corticosteroids, which were previously responsible for the remission of these conditions. Currently she is in her third year post surgery, after achieving cure of Cushing's syndrome, she remains hypocortisolaemic requiring steroids replacement in the form of hydrocortisone, as a result of persistent prolonged hypercortisolaemia.

\section{Discussion}

We present a case of non-ACTH-dependent Cushing's syndrome who had delayed diagnosis, despite the presence of several typical features, leading to significant morbidity and life-threatening complications. The main reason for the delayed diagnosis was the confounding influence of the previous use of steroids in oral and intra-articular forms. It was only when the details of the precise dose and duration of the use of steroids was elicited, the discordance between the florid clinical signs and the steroid use was identified and the diagnosis was considered. The presence of several other long-standing medical conditions and lack of continuity of outpatient review may have contributed to the delay. During the 3-year journey, she had multiple clinic visits (at least 5/ year), 17 chest radiographs (CXRAY), 6 spirometry, 4 HRCTs, 2 echocardiogram, 2 respiratory muscle strength testing, 2 ultrasonography of the chest/abdomen and 1 lung biopsy before the diagnosis was made. 
The most unusual aspect of this case was the presence of significant respiratory muscle and diaphragmatic weakness, which led to progressive and debilitating symptoms and life-threatening acute respiratory failure. She presented with progressive exertional dyspnoea resulting in significantly reduced mobility. She also developed severe respiratory failure triggered by relatively modest lower respiratory infection requiring level-3 management in an intensive care unit.

Proximal lower-limb girdle myopathy is common in Cushing's syndrome, and classically results in difficulty in rising from a squatting position without assistance, inability to climb stairs or getting up from a low chair (1). Interestingly, in addition to these features our patient experienced respiratory and diaphragmatic muscle weakness secondary to metabolic myopathy; a diagnosis made incidentally during ultrasound abdomen for elevated liver enzymes which prompted further investigations by means of respiratory muscle strength testing.

There are only a few case reports in the literature where patients presented with respiratory insufficiency secondary to hypercortisolism. Blanco et al. reported a case of a 55-year-old woman with severe respiratory insufficiency associated with hypoxaemia, hypercapnea and respiratory acidosis confirmed with restrictive ventilatory failure on spirometry and reduction in MIP and MEP on plethysmography. Her symptoms improved after initiation of ketoconazole (2). Tanaka et al. reported a case of Cushing's syndrome who presented with chronic hypoxemia but unlike in our case, this was secondary to metabolic alkalosis driven by hypokalaemia secondary to hypercortisolism and in her case hypoxemia and metabolic acidosis reversed by normalisation of potassium (3).

Cushing's myopathy is caused the catabolic effects of excess glucocorticoid on skeletal muscle and is amplified by physical inactivity (1). Glucocorticoids have a direct catabolic effect on skeletal muscle and activate an intermediary metabolism that provides amino acids as a substrate for gluconeogenesis. Activation of the glucocorticoid receptor appears to be involved as myopathy can be prevented by a glucocorticoid receptor antagonist $(4,5)$. Hypercortisolism can also lead to myopathy by other indirect mechanisms. It can interfere with insulin-like growth factor-1 (IGF-1) signalling, leading to increased myocyte apoptosis (6), which leads to myopathy and muscle atrophy. An intracellular signalling molecule with protein kinase activity known as Akt 1 may play a central role in the atrophic and hypertrophic responses of muscle to glucocorticoids and IGF-1, respectively $(7,8,9)$. Suppression of the Akt 1 causes an increase of ubiquitin-ligase atrogin-1 production that targets muscle protein degradation. Glucocorticoids also lead to reduction in muscle differentiation. It occurs secondary to enhanced degradation through ubiquitination of MyoD, which is a master transcriptional switch for muscle development and regeneration (5).

The diagnosis of glucocorticoid (GC)-induced myopathy can be made by clinical, laboratory and electrophysiological criteria (10). Typical electromyogram (EMG) findings include increased number of low amplitude, short duration and at times polyphasic, motor unit potentials (11). On the other hand, EMG, can often be normal, as was the case with our patient (11). Furthermore, diagnosis can also be made using muscle biopsy and MRI, although in the latter case, the method does not distinguish idiopathic inflammatory myopathy from steroid myopathy (12). As in our patient, plethysmography is another method, which may reveal restrictive respiratory failure.

The main treatment consists of treating the underlying cause that is hypercortisolism. Various medications including creatine, branched amino acids, androgens, vitamin supplements and recombinant IGF-1 have been suggested to hinder the development of GC-induced myopathy, but their effects are poorly analysed and in some the expediency of these approaches in clinical practice is questionable $(13,14)$. Non-pharmacological treatment are also advisable and these include physical exercise programmes including moderate aerobic, resistance and stretching exercises to reverse or mitigate muscle wasting and improve mobility. None of these would have been applicable in our patient in view of significant rheumatoid arthritis and overall debility $(14,15)$. Nevertheless, high-intensity exercises should be avoided as they can be harmful to the muscles (16). In our patient, further confirmation and localisation investigation revealed evidence of a functioning left adrenal tumour. Removal of the tumour led to a prompt improvement of muscle strength as evidenced by improvements in readings in the pulmonary function tests and respiratory muscle strength test.

Sedentary lifestyle may increase the risk of muscle weakness in a patient taking corticosteroids, since corticosteroids seem to affect less active muscles preferentially unlike in this case where more active respiratory muscles were affected. This may be due to the fact that our patient has an underlying history of rheumatoid arthritis which due to illness, there may be a high amount of dormant muscle hence leading to widespread myopathy. 


\section{Conclusion}

Cushing's syndrome usually affects proximal muscle and several mechanisms have been proposed. Due to the unusual presentation (breathlessness) it was challenging for non-endocrine specialties to consider Cushing's syndrome and to treat appropriately. Though delay in diagnosis, patient was very satisfied in the end as her breathlessness improved after treatment.

\section{Declaration of interest}

The authors declare that there is no conflict of interest that could be perceived as prejudicing the impartiality of this case report.

\section{Funding}

This research did not receive any specific grant from any funding agency in the public, commercial or not-for-profit sector.

\section{Patient consent}

The written informed consent has been obtained from the patient for publication of the submitted article and accompanying images.

\section{Author contribution statement}

L Kandaswamy writing and gathered clinical data. C Gherman-Ciolac writing and gathered clinical data. J Mann is the Consultant Respiratory Physician managing the case prior to referral to the Endocrinology team. $\mathrm{H} \mathrm{N}$ Buch is the Consultant Endocrinologist managing the case, reviewed and commented on the manuscript.

\section{References}

1 Ferrando AA, Stuart CA, Sheffield-Moore M \& Wolfe RR. Inactivity amplifies the catabolic response of skeletal muscle to cortisol. Journal of Clinical Endocrinology and Metabolism 199984 3515-3521.

2 Blanco C, Marazuela M, Flores J \& Alvarez J. Severe respiratory failure secondary to Cushing's myopathy. Journal of Endocrinological Investigation 200124 618-621. (https://doi.org/10.1007/BF03343903)

3 Tanaka M, Yano T, Ichikawa Y \& Oizumi K. A case of Cushing's syndrome associated with chronic respiratory failure due to metabolic alkalosis. Internal Medicine 199931 385-390. (https://doi. org/10.2169/internalmedicine.31.385)
4 Konagaya M, Bernard PA \& Max SR. Blockade of glucocorticoid receptor binding and inhibition of dexamethasone-induced muscle atrophy in the rat by RU38486, a potent glucocorticoid antagonist. Endocrinology 1986119 375-380. (https://doi.org/10.1210/endo-119$1-375)$

5 Sun L, Trausch-Azar JS, Muglia LJ \& Schwartz AL. Glucocorticoids differentially regulate degradation of MyoD and Id 1 by N-terminal ubiquitination to promote muscle protein catabolism. PNAS 2008 105 3339-3344. (https://doi.org/10.1073/pnas.0800165105)

6 Singleton JR, Baker BL \& Thorburn A. Dexamethasone inhibits insulin-like growth factor signaling and potentiates myoblast apoptosis. Endocrinology 2000141 2945-2950. (https://doi. org/10.1210/endo.141.8.7621)

7 Hoffman EP \& Nader GA. Balancing muscle hypertrophy and atrophy. Nature Medicine 200410 584-585. (https://doi.org/10.1038/ nm0604-584)

8 Sandri M, Sandri C, Gilbert A, Skurk C, Calabria E, Picard A, Walsh K, Schiaffino S, Lecker SH \& Goldberg AL. Foxo transcription factors induce the atrophy-related ubiquitin ligase atrogin-1 and cause skeletal muscle atrophy. Cell 2004117 399-412. (https://doi. org/10.1016/S0092-8674(04)00400-3)

9 Stitt TN, Drujan D, Clarke BA, Panaro F, Timofeyva Y, Kline WO, Gonzalez M, Yancopoulos GD \& Glass DJ. The IGF-1/PI3K/Akt pathway prevents expression of muscle atrophy-induced ubiquitin ligases by inhibiting FOXO transcription factors. Molecular Cell 2004 14 395-403. (https://doi.org/10.1016/S1097-2765(04)00211-4)

10 Pereira RM \& Freire de Carvalho J. Glucocorticoid-induced myopathy. Joint Bone Spine 201178 41-44. (https://doi.org/10.1016/j. jbspin.2010.02.025)

11 Minetto MA, Lanfranco F, Botter A, Motta G, Mengozzi G, Giordano R, Picu A, Ghigo E \& Arvat E. Do muscle fiber conduction slowing and decreased levels of circulating muscle proteins represent sensitive markers of steroid myopathy? A pilot study in Cushing's disease. European Journal of Endocrinology 2011164 985-993. (https:// doi.org/10.1530/EJE-10-1169)

12 Lovitt S, Marden FA, Gundogdu B \& Ostrowski ML. MRI in myopathy. Neurologic Clinics 200422 509-538. (https://doi. org/10.1016/j.ncl.2004.03.008)

13 Pereira RM \& Freire de Carvalho J. Glucocorticoid-induced myopathy. Joint Bone Spine 201178 41-44. (https://doi.org/10.1016/j. jbspin.2010.02.025)

14 Minetto MA, Lanfranco F, Motta G, Allasia S, Arvat E \& D'Antona G. Steroid myopathy: some unresolved issues. Journal of Endocrinological Investigation 201134 370-375. (https://doi.org/10.1007/ BF03347462)

15 Falduto MT, Czerwinski SM \& Hickson RC. Glucocorticoid-induced muscle atrophy prevention by exercise in fast-twitch fibers. Journal of Applied Physiology 199069 1058-1062. (https://doi.org/10.1152/ jappl.1990.69.3.1058)

16 Uchikawa K, Takahashi H, Hase K, Masakado Y \& Liu M. Strenuous exercise-induced alterations of muscle fiber cross-sectional area and fiber-type distribution in steroid myopathy rats. American Journal of Physical Medicine and Rehabilitation 200887 126-133. 